\title{
CME Infectious diseases (113044) self-assessment questionnaire
}

\author{
Edited by Jon Friedland and Tahseen A Chowdhury
}

SAQs and answers are ONLINE for RCP fellows and collegiate members

\section{Format}

Candidates are asked to choose the best answer from the five possible answers. This best of five format is used in many medical examinations; however, the questions are not intended to be representative of those used in the MRCP(UK) Part 1 or Part 2 Written Examinations.

The answering process

1 Go to www.rcplondon.ac.uk/SAQ

2 Log on using your usual RCP username and password

3 Select the relevant $\mathrm{CME}$ question paper

4 Answer all 10 questions by selecting the best answer from the options provided

5 Once you have answered all the questions, click on Submit

\section{Registering your external CPD credits}

Carrying out this activity allows you to claim two external CPD credits. These will be automatically transferred to your CPD diary, where you can review the activity and claim your points.

1. Which of the following is a clinical indicator of sepsis using the q(quick)SOFA tool?
(a) $\mathrm{CRP}>50$
(b) Neutrophil count $>10 \times 10^{3} / \mathrm{mL}$
(c) Pulse greater than 100
(d) Respiratory rate $>22$
(e) Temperature $>38^{\circ} \mathrm{C}$

2. Which of the following would be appropriate immediate steps for a patient admitted to hospital with sepsis?
(a) Blood cultures
(b) CT brain
(c) Immediate transfer to a critical care unit
(d) Maintenance of blood sugar $<6 \mathrm{mM}$
(e) Resuscitation with human albumin

3. Which of the following is a characteristic feature of sepsis?

(a) Elevated amylase

(b) Extensive tissue necrosis (c) Increased cardiac ejection fraction

(d) Increased systemic vascular resistance

(e) Prolonged clotting times

4. A 49-year-old man is admitted with a low-grade temperature, an acutely inflamed knee joint and raised inflammatory markers. Blood cultures are taken and an ultrasound-guided synovial aspirate performed, before antibiotics are administered. He has an arthroscopic washout of the joint and pus is found. Microscopy shows 100,000 leucocytes $/ \mathrm{mm}^{3}$. Gram stain is negative and cultures after 48 hours show no growth. Which of the following is NOT important to consider.

(a) Taking a sexual history and to consider a urethral swab or urine NAAT testing, rectal/throat swabs and 16S PCR testing on synovial fluid

(b) Microscopic examination of synovial fluid under polarised light

(c) Taking a travel and diet history and to prolong cultures for Brucella spp if relevant

(d) Taking a throat swab for Staphylococcus aureus and Betahaemolytic streptococci

(e) Taking a travel and tick bite exposure history and consider Lyme serology

5. A 75-year-old woman presents with a 3-day history of fever and lower back pain. There are no neurological findings on examination. MRI shows acute discitis at L1/2 level. Which is the LEAST likely causative organism?
(a) Staphylococcus aureus
(b) Bacteroides fragilis
(c) Streptococcus spp
(d) Escherischia coli
(e) Proteus spp

6. In which of these patients would it be reasonable to proceed with lumbar puncture without first obtaining brain imaging?

(a) A 30-year-old man with a temperature of $38.5^{\circ} \mathrm{C}$ and fall in conscious level (GCS now 11/15) within the past 24 hours

(b) A 62-year-old woman with fever and personality change who is mildly confused (GCS of 14/15) now

(c) A 43-year-old man with a history of intravenous drug use and HIV infection with fever, headache and new left-sided weakness over the last 24 hours 
(d) A 29-year-old woman with headache and double vision. Examination shows a new eye movement abnormality (difficulty abducting the left eye) although GCS is 15

(e) A 19-year-old woman with drowsiness, a new severe global headache over the last 7 days and optic disc swelling on fundoscopy

7. A 57 -year-old man with type 2 diabetes mellitus is admitted with a 2-day history of a painful swollen right lower leg. He was febrile, but had no features of shock or organ dysfunction on admission and no skin breaks, subcutaneous collections or history of trauma. FBC U\&E and LFTs were within normal range. He has never been admitted to hospital before. He was commenced on flucloxacillin $2 \mathrm{~g} 6$-hourly IV in the Emergency Department. After 24 hours in hospital he spikes a temperature of $38.5^{\circ} \mathrm{C}$. His leg is largely clinically unchanged and he remains haemodynamically stable. What is the most appropriate management step?
(a) Addition of clindamycin IV
(b) Continue to observe for a further 24 hours prior to altering antimicrobial therapy
(c) Addition of vancomycin
(d) Addition of oral prednisolone
(e) Escalation of antimicrobials to piperacillin-tazobactam

8. A 72-year-old retired school teacher is referred to the Emergency Department with a painful hot left lower leg. She had attended her GP a week ago, was diagnosed with cellulitis and treated with clarithromycin $500 \mathrm{mg}$ bd po for 5 days. She reports that her leg has continued to deteriorate and she has developed fevers, chills and anorexia. She has no past medical history, no drug allergies and clinical exam reveals only an inflamed left lower leg with no purulence. What is the most appropriate antimicrobial therapy?
(a) Vancomycin
(b) Clindamycin
(c) Metronidazole
(d) Flucloxacillin
(e) Ceftriaxone

9. A 63-year-old woman with a history of left leg lymphoedema secondary to previous inguinal node clearance and radiotherapy presents with her third episode of left leg cellulitis. Her infection has responded to flucloxacillin on each previous occasion. In addition to flucloxacillin on this occasion what other therapies may be appropriate?
(a) Benzylpenicillin IV for 7 days
(b) Vancomycin
(c) Long term oral penicillin
(d) A supply of clindamycin to have at home
(e) IV IgG

10. Which of the following statements is correct regarding acute meningitis?
(a) Dexamethasone should never be commenced until antibiotics have been administered
(b) In Listeria meningitis a 2-week course of antibiotics should be given
(c) In fungal meningitis the CSF white cell count is typically elevated, protein is normal or moderately elevated and glucose is normal
(d) Patients with viral meningitis often report problems post discharge
(e) Herpes simplex meningitis should be treated with aciclovir

\section{CME Acute medicine SAQ}

Answers to the CME SAQ published in Clinical Medicine in February 2018

$\begin{array}{llllllllll}\text { Q1 } & \text { Q2 } & \text { Q3 } & \text { Q4 } & \text { Q5 } & \text { Q6 } & \text { Q7 } & \text { Q8 } & \text { Q9 } & \text { Q10 } \\ \text { (b) } & \text { (d) } & \text { (e) } & \text { (c) } & \text { (c) } & \text { (e) } & \text { (d) } & \text { (d) } & \text { (c) } & \text { (a) }\end{array}$

